\title{
An Alternative Form of Laser Beam Characterization
}

\author{
G.A. Knorovsky \& D.O. MacCallum \\ Sandia National Laboratories, Albuquerque, NM 87185-0367
}

\begin{abstract}
Careful characterization of laser beams used in materials processing such as welding and drilling is necessary to obtain robust, reproducible processes and products. Recently, equipment and techniques have become available which make it possible to rapidly and conveniently characterize the size, shape, mode structure, beam quality $\left(\mathrm{M}^{2}\right)$, and intensity of a laser beam (incident power/unit area) as a function of distance along the beam path. This facilitates obtaining a desired focussed spot size and also locating its position. However, for a given position along the beam axis, these devices typically measure where the beam intensity level has been reduced to $1 / \mathrm{e}^{2}$ of maximum intensity at that position to determine the beam size. While giving an intuitive indication of the beam shape since the maximum intensity of the beam varies greatly, the contour so determined is not an iso-contour of any parameter related to the beam intensity or power. In this work we shall discuss an alternative beam shape formulation where the same measured information is plotted as contour intervals of intensity. We shall also discuss how this technique may be useful in laser beam materials processing.
\end{abstract}

\section{Introduction}

Why do we want to characterize laser beams? In an engineering setting, it's because we want them to do something in a reproducible manner. If we have a product/process that we expect to fulfill specific requirements, we certainly don't just turn on the laser and starting welding, cutting, heat treating, etc. without carefully verifying that all the machine settings are correct. Even so, sometimes things don't quite work out, as perhaps the laser head was bumped or some component has started to degrade. Thus we need to know that our primary process tool, the laser beam, is just the way it was originally set with respect to laser power distribution versus time and space. In a scientific setting, we need to know this same information in order to discern a quantitative relation between the cause and effect we are studying.

Past and present methods of beam characterization have ranged from the "quick and dirty" (what size hole does the beam make in a plastic sheet or cube) to extremely sophisticated (commercial instruments automatically measuring the beam power in a plane and the caustic of the beam "size" versus propagation distance). They also vary depending upon whether the focused or unfocused beam is being examined. Several techniques and the parameter being measured are summarized in Table 1.

Recently an ISO standard [1] for characterizing laser beams has emphasized the use of the $\mathrm{M}^{2}$ parameter to determine beam quality. The equipment required to determine this parameter is complex (particularly for pulsed beams), but the information gained is also quite comprehensive. While this method is highly recommended, we have found that by using the information gained in determining $\mathrm{M}^{2}$ and replotting it in a slightly 


\section{DISCLAIMER}

This report was prepared as an account of work sponsored by an agency of the United States Government. Neither the United States Government nor any agency thereof, nor any of their employees, make any warranty, express or implied, or assumes any legal liability or responsibility for the accuracy, completeness, or usefulness of any information, apparatus, product, or process disclosed, or represents that its use would not infringe privately owned rights. Reference herein to any specific commercial product, process, or service by trade name, trademark, manufacturer, or otherwise does not necessarily constitute or imply its endorsement, recommendation, or favoring by the United States Government or any agency thereof. The views and opinions of authors expressed herein do not necessarily state or reflect those of the United States Government or any agency thereof. 


\section{DISCLAIMER}

Portions of this document may be illegible in electronic image products. Images are produced from the best available original document. 
Table 1. Some Methods of Beam Characterization

\begin{tabular}{|c|c|c|}
\hline Method & Parameter Measured & Comments \\
\hline Burn paper & Diameter, Modes & Unfocused beam \\
\hline Fluorescent Screen/ UV & Diameter & Unfocused beam \\
\hline Kapton film/metal foil & Diameter, Focal location & Focused beam \\
\hline $\begin{array}{c}\text { Acrylic Block } \\
\text { hollipop (Thermocouple in } \\
\text { heat sink block) }\end{array}$ & Diameter, Modes & Unfocused beam \\
\hline Photodiode & Energy & Unfocused beam \\
\hline $\begin{array}{c}\text { Diamond } \\
\text { Apertures/Integrating }\end{array}$ & $\begin{array}{c}\text { Power versus time, Energy } \\
\text { Diameter }\end{array}$ & Small fraction of raw beam \\
\hline $\begin{array}{c}\text { Moving slit/photodiode } \\
\text { Flying wire/photodiode }\end{array}$ & $\begin{array}{c}\text { Intensity profile (Modes), } \\
\text { Diameter, Energy }\end{array}$ & $\begin{array}{c}\text { Intensity profile (Modes), } \\
\text { Diameter, Energy }\end{array}$ \\
\hline $\begin{array}{c}\text { Detector Arrays } \\
\text { with/without Neutral ber } \\
\text { Density filters }\end{array}$ & $\begin{array}{c}\text { Intensity profile (Modes), } \\
\text { Diameter, Energy }\end{array}$ & Focused beam \\
\hline
\end{tabular}

different manner, we gain increased understanding of the beam-material interaction region.

\section{Experimental}

The basis for the $\mathrm{M}^{2}$ parameter is the beam propagation equation [2] (CW case, i.e., temporal dependence ignored, radial symmetry also assumed for simplicity):

$$
\omega(z)=\left(\omega_{0}^{2}+\left(M^{2} \lambda\left(z-z_{0}\right) / \pi \omega_{0}\right)^{2}\right)^{0.5}
$$

where $\omega(z)$ is the radius of the beam as a function of distance along the propagation direction and $\lambda$ is the laser wavelength. The minimum waist and location of the beam are represented by $\omega_{0}\left(z_{0}\right)$, where $z$ 's origin is at the focussing lens plane. Thus information on the beam size as a function of beam propagation distance must be determined and this parabolic equation fit to the data, with $\mathrm{M}^{2}, \mathrm{Z}_{0}$ and $\omega_{0}$ acting as fit parameters. The size of the beam is determined by the radius of a circle within which $1-\left(1 / e^{2}\right)$ of the total beam energy (or power) is contained. The results of such an analysis of data obtained on a Prometec beam scanning profilometer (which uses a rotating wire with small aperture to sample the focussed beam) are shown in Figure 1, for four different laser beam powers on a CW Nd:YAG system with hard optics beam delivery. Curve fitting and plotting was done using Kaleidagraph [3] software.

While the lines indicating the beam radius versus propagation distance in Figure 1 (hereafter referred to as beam caustics) are indicative of the energy distribution, they are misleading in that the actual value of the laser beam irradiance(or intensity, which has units of W/area) on these lines varies strongly as a function of $z$ This is explained as follows. Given that the integrated power is constant along the beam (i.e. there is no 
attenuation), and that the beam intensity profile has the same functional shape (e.g. Gaussian), then the ratio of the the intensity at the $1-\left(1 / \mathrm{e}^{2}\right)$ position to the peak intensity will be contant. Since the peak intensity varies along $z$, the value of intensity at the $1-$ $\left(1 / \mathrm{e}^{2}\right)$ position must also vary. Since it is the intensity of the beam that controls how it

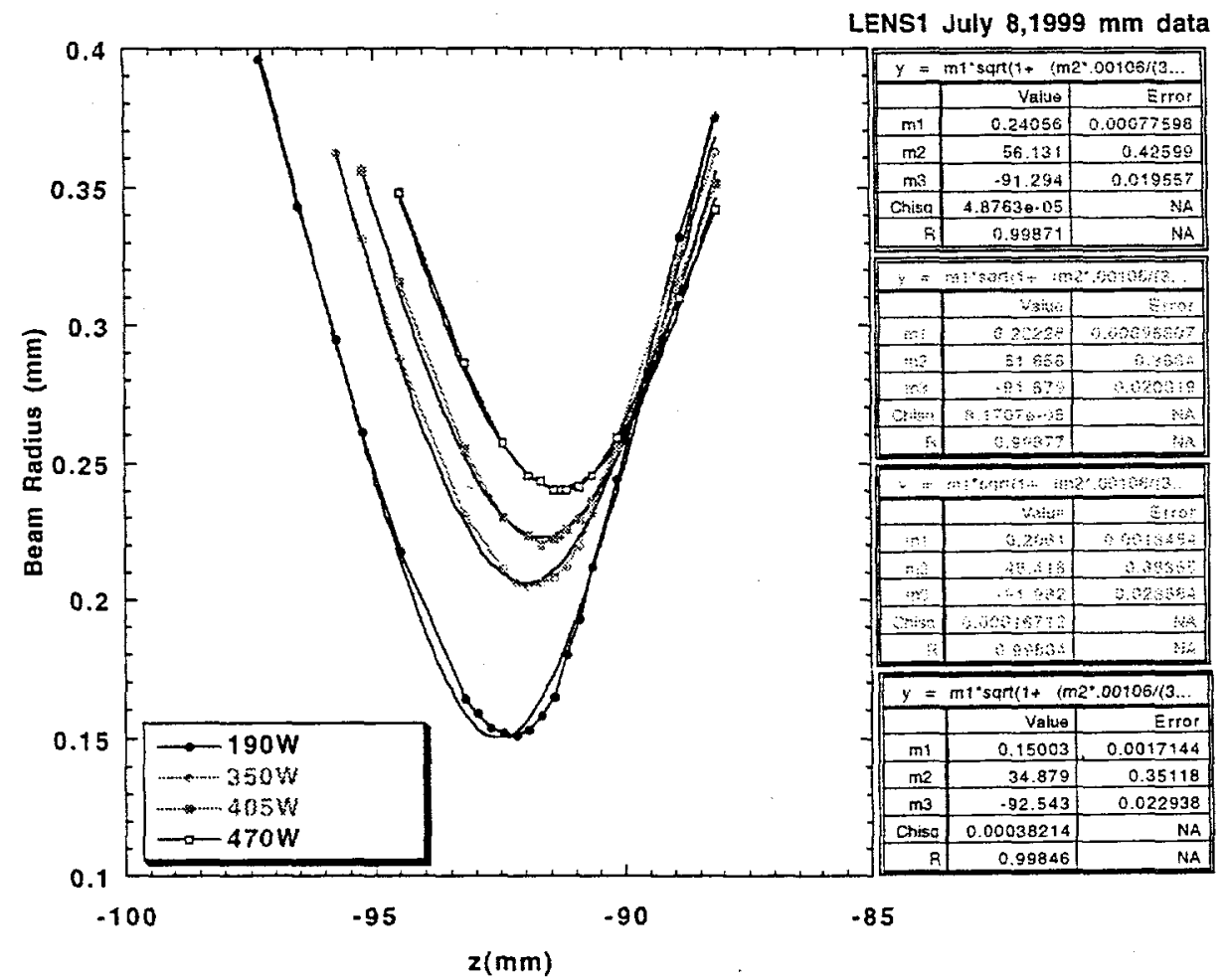

Figure 1: $\mathrm{M}^{2}$ and beam caustic versus power (in W) for US Laser $500 \mathrm{~W}$ Nd:YAG CW laser, hard optic beam delivery system. The four tables are arranged from highest to lowest power in top-to-bottom order; $\mathrm{m} 1=$ minimum beam radius $\omega_{0}, \mathrm{~m} 2=\mathrm{M}^{2}, \mathrm{~m} 3=\mathrm{z}_{0}$, Chisq and $\mathrm{R}$ are statistical parameters measuring the goodness of fit to the data.

interacts with matter, this strong variation is a serious shortcoming; and hence, the caustics give a poor measure of the beam's "size". Typically, the beam intensity in a plane at a given $z$ location may also be plotted with the present generation of beam profilers. This gives a very clear representation of the intensity distribution of the beam [4]. Unfortunately, at least for the contemporary beam profiling systems we are familiar with, it is not possible to combine these plots along the beam. Since we felt that these contour plots at one location along the beam are the clearest representation of the beam intensity available, we considered that a contour plot of the beam intensity versus radius and propagation distance would be even more clearly indicative of a beam's potential for interaction with matter.

To implement this technique we proceeded as follows: first, for simplicity we assumed a laser beam with an exponential shape of beam intensity versus radius, which allowed us to relate peak intensity to the total power in the beam and beam size via [5]:

$$
I_{0}(z)=2 P / \pi \omega(z)^{2}
$$


The beam's radius $\omega(\mathbf{z})$ was described by the previously given beam propagation equation with given values of $\mathrm{M}^{2}, \mathrm{z}_{0}$ and $\omega_{0}$ found experimentally. Next, we calculated intensity versus $r$ and $z$ :

$$
I(r, z)=I_{0}(z) \exp -2(r / \omega)^{2}
$$

Finally, we contour plotted the I(r, z) using MATLAB [6], giving plots shown in Figure 2.

\section{Results \& Discussion}

Some observations may be made from a comparison of Figures 1 and 2. The first observation concerns the shape of the beam. The caustic is basically parabolic, whereas the intensity contours form closed curves. As already noted, the intensity along the caustic varies; the upper left panel of Figure 2 indicates this, as the caustic line away from the minimum waist is nearly perpendicular to the contours. The second observation concerns the size of the beam. The caustic minimum waist "size" appears nearly equivalent to the lowest contour of intensity (simply because it was 0.2 times the peak value, as compared with $1 / \mathrm{e}^{2}=0.14$ ), and indeed, this contour increases its extent as the beam power increases. However, the highest intensity contour did not change much at all (except in $\mathbf{z}$ position), implying that the "sweet spot" of the beam was relatively unaffected by the average power. This would certainly be of great interest for controlling a process that is limited by a threshold intensity. Another observation may be made regarding the depth of focus of the beam. The caustic curve's rapid divergence from the minimum waist position correctly implies that the depth of focus is small; however, the extent of the lowest intensity contour would imply that the depth of focus is quite a bit larger, at least if this value of intensity (which was nearly equivalent with the caustic curve at the minimum waist) is the controlling factor. Experience from laser welding where the penetration is typically relatively insensitive over distances of $\sim 1 \mathrm{~mm}$ or so seems to indicate that the "sweet spot" intensity contour may control. A caustic curvecalculated to be equivalent to this intensity contour (i.e. one at a value much greater than $\left.1-\left(1 / \mathrm{e}^{2}\right)\right)$ would diverge much more slowly and hence the caustic curve predictions are only qualitative at best.

As another example of how the intensity contour representation is useful, we compare the beam intensities of two lasers with differing beam delivery systems. We showed a hard optic delivery system in Figure 2. As an example of a fiber-optic delivery system, we show in Figure 3 the results of a 1200-W CW Nd:YAG from the same manufacturer. Comparing the two at nearly equivalent power settings in Figure 4, it can be seen that while both have about the same minimum waist diameter, the fiber optic delivery system has a reduced peak power but an increased depth of focus. Also, in contrast to the data of Figure 2, this laser's "sweet spot" does grow with increased average power.

If one were faced with the problem of transferring a given process from one laser to the other, it is not clear that it could be done without completely redeveloping the schedule because of the changes in beam intensity, size and distribution. Certainly, the 
tolerances with respect to focus would have to be redefined, as would the average power settings.

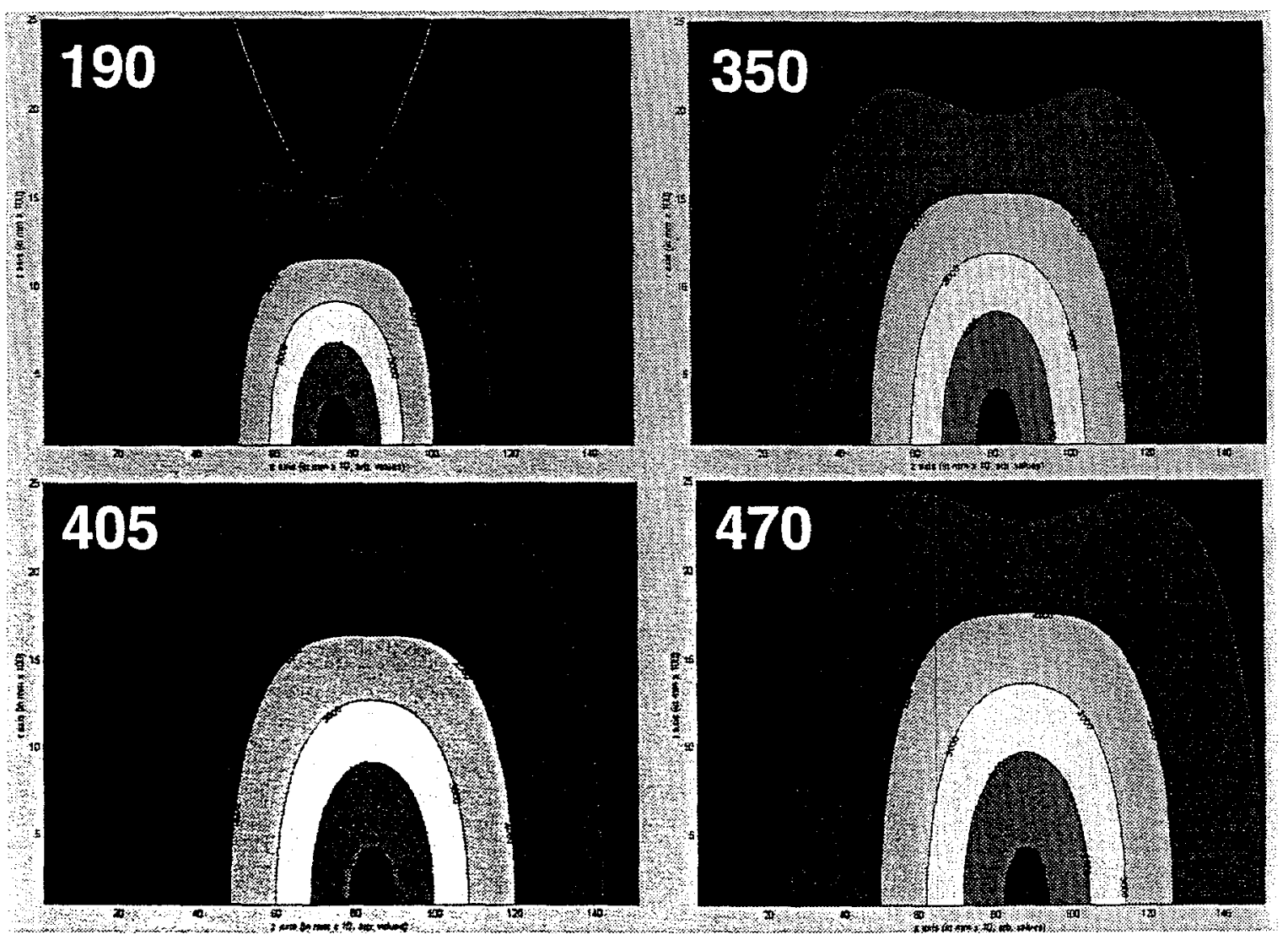

Figure 2: Alternative plot of intensity contours versus radius and beam propagation distance derived from data obtained from Figure 1. The average power in Watts is noted on each panel. Data for 190 Watts also includes a plot of the caustic "size" versus $Z$. The horizontal ( $z$ ) and vertical ( $r$ ) scales are the same for all four panels. Full scale for the vertical axis is $0.25 \mathrm{~mm}$; for the horizontal axis it is $2.5 \mathrm{~mm}$ (note that the vertical scale is expanded $10 \mathrm{x}$ relative to the horizontal). Intensity contours are in intervals of 1 $\mathrm{kW} / \mathrm{mm}^{2}$, with the outermost (lowest intensity) equal to $1 \mathrm{~kW} / \mathrm{mm}^{2}$.

\section{Conclusions}

We have presented an alternative and simple form of beam characterization: contour plotting intensity versus radial and beam propagation directions. Such plotting requires no additional information beyond that obtained from modern beam profilers but adds insight about the spatial extent of a laser beam and how this may affect beammaterial interactions. In future work we would like to relax the Gaussian beam assumption made here and instead directly access intensity versus position data from the files generated by the beam profiler in order to provide a true three dimensional representation of the beam intensity profile in space. 


\section{Acknowledgments}

Sandia is a multiprogram laboratory operated by Sandia Corporation, a Lockheed Martin Company, for the United States Department of Energy under Contract DE-AC0494AL85000.

\section{References}

1. Test methods for laser beam parameters: Beam widths. divergence angle and beam propagation factor, Document ISO/11146. ISO, Nov. 1993.

2. Siegman. A.E., "New Developments in Laser Resonators". Optical Resonators, SPIE. Los Angeles. CA. 1990, pp 2-14.

3. Kaieidagraph. Synergy Software, Reading, PA, 1999.

4 . Roundy. C.B. "Current Technology of Laser Beam Profile Measurements", downioadable from ww spricon.com, 1999.

5. Ready, J.F. Industrial Applications of Lasers, Academic Press, New York, 1978, p41.

6. MATLAB. The MathWorks. Inc, Natick, MA. 1999.

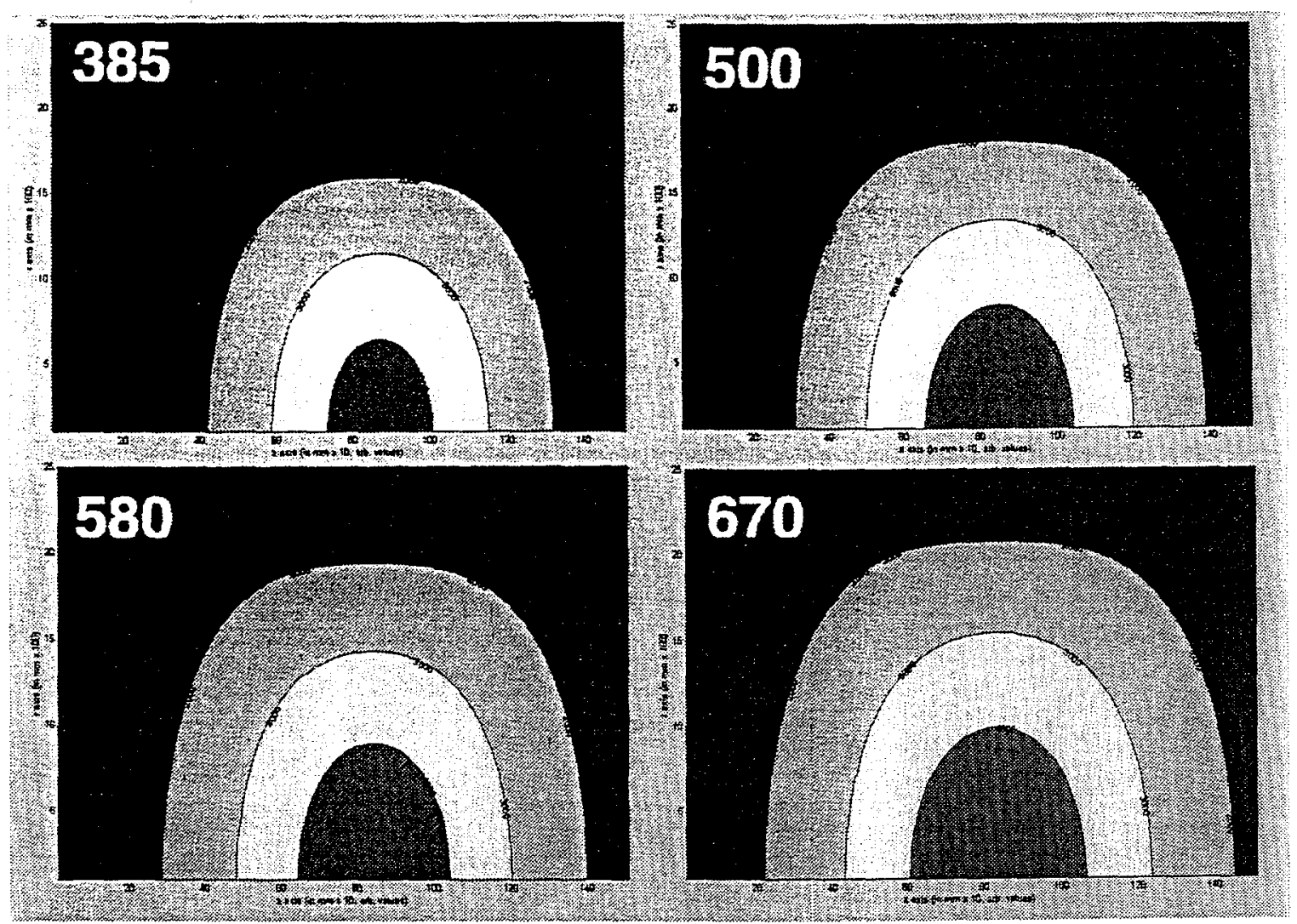

Figure 3: Intensity contours versus Power for a fiber-optic delivery, 1200-W Nd:YAG laser. The horizontal ( $\mathbf{z}$ ) and vertical (r) scales are the same for all four panels. Full scale for the vertical axis is $0.25 \mathrm{~mm}$; for the horizontal axis it is $2.5 \mathrm{~mm}$ (note that the vertical scale is expanded $10 \mathrm{x}$ relative to the horizontal). Intensity contours are in intervals of 1 $\mathrm{kW} / \mathrm{mm}^{2}$, with the outermost (lowest intensity) equal to $1 \mathrm{~kW} / \mathrm{mm}^{2}$. 

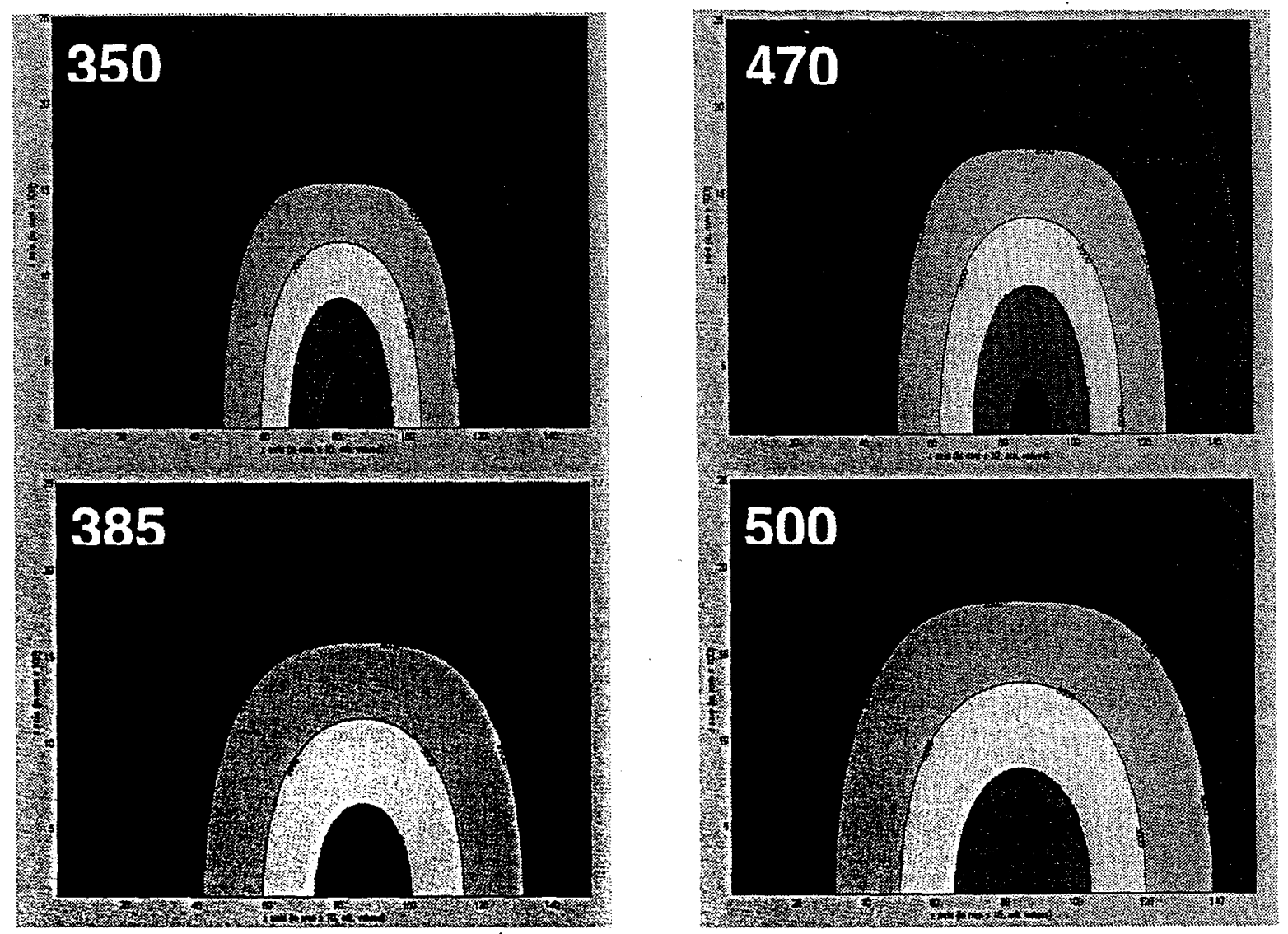

Figure 4: Intensity contours at nearly equivalent power levels for the two lasers. Top panels from $500-W$, hard optic system, bottom from $1200-W$, fiber optic system. The horizontal ( $z$ ) and vertical ( $r$ ) scales are the same for all four panels. Full scale for the vertical axis is $0.25 \mathrm{~mm}$; for the horizontal axis it is $2.5 \mathrm{~mm}$ (note that the vertical scale is expanded $10 \mathrm{x}$ relative to the horizontal). Intensity contours are in intervals of 1 $\mathrm{kW} / \mathrm{mm}^{2}$, with the outermost (lowest intensity) equal to $1 \mathrm{~kW} / \mathrm{mm}^{2}$.

\section{Meet the Authors}

Gerald Knorovsky is a Senior Member of Technical Staff at Sandia National Laboratories, Albuquerque, NM, in the Joining, Coating and Net Shapes Department. He has a Sc.D. in Physical Metallurgy from the Massachusetts Institute of Technology, and has worked in fields involving metallurgy, joining, fracture, process analysis and modelling for over twenty years. He is a member of the Laser Institute of America, ASM International and the American Welding Society.

Danny MacCallum is a Principal Technologist at Sandia National Laboratories, Albuquerque, NM, in the Joining, Coating and Net Shapes Department. He has a B.Sc. Degree in Applied Mathematics and Physics from the University of New Mexico and eleven years experience in data acquisition, analysis and numerical computing. $\mathrm{He}$ is a member of the Laser Institute of America and the American Welding Society. 